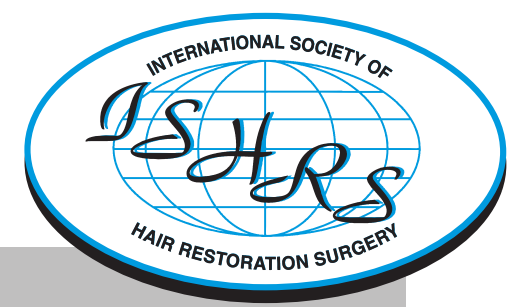

\section{Inside this issue}

President's Message ...

Co-editors' Messages...

Notes from the Editor Emeritus:

Dow B. Stough, MD

Controversies:

Where Business Meets Medicine...

Human Recombinant Hyaluronidase as an Adjunct in Donor Strip Harvesting...

Part 2: LLLT Devices, Medical Device Regulation, and Impact on Development.

Hair's the Question: FFA.

Ask the Fellows

Complications \& Difficult Cases: Recipient Site Infection Following a Hair Transplantation Procedure.....20 Cyberspace Chat:

Post-operative Nausea and Vomiting

Associated with Anesthesia During Hair

Transplantation...

Review of the Literature.

2014 Annual Meeting Poster

Winners: Second Place

Meetings \& Studies:

Review of the 22nd ASM of

the ISHRS.

Regional Societies Profiles:

Polish Society of Hair

Restoration Surgery.

Letter to the Editor

Messages from the 2015 ASM

Program Chairs

Classified Ads.

\section{CALL FOR ABSTRACTS}

www.ishrs.org/AnnualMeeting.html

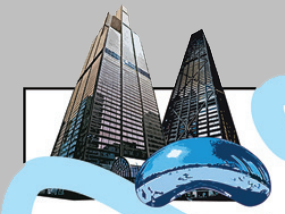

CHICAGO'15 ISHRS 23RD ANNUAL
SCIENTIFIC MEETING SEPTEMBER 9-13

Submission Deadline: February 11, 2015

\title{
Female Androgenetic (?) Alopecia
}

\author{
Andrew Messenger, MBBS Sheffield, England, UK, Silke Redler, PhD Bonn, Germany, \\ Regina C. Betz, MD Bonn, Germany a.g.messenger@sheffield.ac.uk
}

It is nearly 100 years since the publication of Dorothy Osborn's paper on the inheritance of common balding. ${ }^{1}$ From a study of 22 families, she concluded that balding is due to a single gene and is inherited as an autosomal dominant trait in men and autosomal recessive in women. Two twin studies, one in young men and the other in elderly men, have confirmed the importance of genetics in male balding showing heritability in the region of $80-95 \%$, ${ }^{2,3}$ although the single gene idea has now been supplanted by a polygenic model. Initial case control studies found an association between male AGA and polymorphisms within the androgen receptor gene on the X chromosome. ${ }^{4}$ The presence of a major AGA locus within the androgen receptor/ectodysplasin A2 receptor region has been confirmed in subsequent genome wide association studies (GWAS) 5 and these studies have gone on to identify a further 11 loci scattered across the genome that also show association with male AGA. ${ }^{6-8}$ The functional significance of these loci is not yet known but may include the regulation of androgen responses and participation in WNT signaling.

What about female AGA? The application of the term "androgenetic" to female hair loss has implied an identity with male AGA, but it has also colored our thinkingbecause we have given it the same name it must be the same condition. Is this really true? There are certainly similarities; like male AGA the

Ectodysplasin A2 Receptor: This is an isoform of ectodysplasin encoded by the ectodermal dysplasia gene. It is an integral component in epidermal and embryonic development and cell differentiation. Variants in EDA2R have been linked to the AR receptor role in androgenetic alopecia.

GWAS: Genome-wide association study is an examination of many common genetic variants in different individuals to see if any variant is associated with a trait.

WNT: WNT protein is a family of signaling molecules that regulate cell to cell interactions during embryogenesis. Abnormalities in these pathways have been linked to a number of clinical conditions.

female form is common

and increases in prevalence and severity in the population with advancing age, and the histopathology of male and female AGA is essentially indistinguishable. Androgens are clearly required for the expression of male AGA and there is little doubt that hyperandrogenism in women, particularly when severe, is associated with scalp hair loss. Hamilton refers to reports of such cases in his seminal publication on the role of testosterone in causing male AGA. ${ }^{9}$ On the other hand, many women with AGA have no other clinical or biochemical signs of hyperandrogenism and female AGA has been reported in the absence of circulating androgens and in androgen insensitivity syndrome. ${ }^{10,11}$ A recent questionnaire study in women being treated with testosterone implants for androgen deficiency actually reported an improvement in hair growth in those complaining of hair thinning prior to treatment. ${ }^{12}$ Antiandrogens have been widely used to treat female AGA but the quality of evidence for efficacy is poor. Of the better studies, a randomized clinical trial (RCT ) of finasteride $1 \mathrm{mg}$ daily in postmenopausal women with AGA failed to show any benefit after a year of treatment. ${ }^{13} \mathrm{~A} 1$-year trial comparing topical minoxidil with cyproterone acetate (CPA) reported improvement in the minoxidil group but overall deterioration in those receiving CPA. ${ }^{14}$ Sub-group analysis did show a small improvement in women with menstrual irregularities taking CPA, possibly suggesting that antiandrogen treatment may work in women with hyperandrogenism. Opinions amongst clinicians treating female AGA do differ and there are those who strongly believe that antiandrogens are effective, but until we get proper RCTs, the controversy is likely to live on. Unfortunately, there is no commercial interest in the field, which makes funding such trials difficult.

Further differences emerge when we explore the genetics of female AGA. The few early studies, such as that of Osborn, assumed male and female AGA share the same genes and provided some evidence that this is the case. However, a twin study in women, although showing evidence for a significant genetic contribution to fronto-temporal recession and to hair graying, found none to hair thinning over the rest of the scalp, implying that hair thinning was non-genetic in origin and presumably had an environmental cause. ${ }^{15}$ This study was conducted 
FAA from front page

in older women but it was performed in the same population and in a similar age group to one of the male twin studies that had shown a strong genetic contribution to male AGA. So far, we have no GWAS in women with AGA. However, using case control methodology, all 12 of the loci known to be associated with male AGA have been tested for an association with female AGA using DNA samples from German and UK cohorts. There was a weak association with the AR/EDA2 locus in the UK patients with early onset hair loss but not the German sample, and no association with any of the other 11 loci in either group (see table below). ${ }^{16-18}$

\begin{tabular}{|ccc|}
\hline \multicolumn{3}{|c|}{ Gene Loci Associations in Pattern Hair Loss } \\
Gene/Locus & Male AGA & FPHL \\
AR/EDA2R & + & $+/-$ \\
20p11 & + & - \\
1p36.22 & + & - \\
$2 \mathrm{q} 35$ & + & - \\
$2 \mathrm{q} 37.3$ & + & - \\
$3 \mathrm{q} 25.1$ & + & - \\
$5 \mathrm{q} 33.3$ & + & - \\
$7 \mathrm{p} 21.1$ & + & - \\
$7 \mathrm{q} 11.22$ & + & - \\
$12 \mathrm{p} 12.1$ & + & - \\
$17 \mathrm{q} 21.31$ & + & - \\
$18 \mathrm{q} 21.1$ & + & - \\
ESR2 & ND & $+/-$ \\
\hline
\end{tabular}

These findings suggest there is some commonality between early onset male and female AGA, but otherwise imply there are significant differences in etiology. Two independent studies in women have suggested a weak association with the estrogen receptor gene ESR $2,{ }^{19,20}$ but, beyond this, we have little evidence for a genetic component to female AGA, in keeping with the results of the twin study. A GWAS will be needed to ascertain whether there are genetic associations in female AGA that are not present in men, but funding such a study has yet to be achieved.

Until we have better evidence for a role for androgens and genetics in female hair loss, the use of "female AGA" should perhaps be abandoned, hence the preference by others and ourselves working in the field for "Female Pattern Hair Loss" as a less committal descriptive term.

\section{References}

1. Osborn, D. Inheritance of baldness. J Hered. 1916; 7:347-355.

2. Nyholt, D.R., et al. Genetic basis of male pattern balding. J Invest Dermatol. 2003; 121:1561-1564.

3. Rexbye, H., et al. Hair loss among elderly men: etiology and impact on perceived age. J Gerontol A Biol Sci Med Sci. 2005; 60:1077-1082.

4. Ellis, J.A., M. Stebbing, and S.B. Harrap. Polymorphism of the androgen receptor gene is associated with male pattern baldness. J Invest Dermatol. 2001; 116:452-455.

5. Richards, J.B., et al. Male-pattern baldness susceptibility locus at 20p11. Nat Genet. 2008; 40:1282-1284.

6. Hillmer, A.M., et al. Susceptibility variants for male-pattern baldness on chromosome 20p11. Nat Genet. 2008; 40:1279-1281.
7. Li, R., et al. Six novel susceptibility loci for early-onset androgenetic alopecia and their unexpected association with common diseases. PLoS Genet. 2012; 8: e1002746.

8. Heilmann, S., et al. Androgenetic alopecia: identification of four genetic risk loci and evidence for the contribution of WNT signaling to its etiology. J Invest Dermatol. 2013; 133:1489-1496.

9. Hamilton, J.B. Male hormone stimulation is prerequisite and an incitant in common baldness. Am J Anat. 1942; 71:451-480.

10. Orme, S., D.E. Cullen, and A.G. Messenger. Diffuse female hair loss: are androgens necessary? Br J Dermatol. 1999; 141:521-523.

11. Cousen, P., and A. Messenger. Female pattern hair loss in complete androgen insensitivity syndrome. Br J Dermatol. 2010; 162:1135-1137.

12. Glaser, R.L., C. Dimitrakakis, and A.G. Messenger. Improvement in scalp hair growth in androgen-deficient women treated with testosterone: a questionnaire study. $\mathrm{Br}$ J Dermatol. 2012; 166:274-278.

13. Price, V.H., et al. Lack of efficacy of finasteride in postmenopausal women with androgenetic alopecia. J Am Acad Dermatol. 2000; 43:768-776.

14. Vexiau, P., et al. Effects of minoxidil $2 \%$ vs. cyproterone acetate treatment on female androgenetic alopecia: a controlled, 12-month randomized trial. Br J Dermatol. 2002; 146:992-999.

15. Gunn, D.A., et al. Why some women look young for their age. PLoS One. 2009; 4: e8021.

16. Redler, S., et al. Investigation of the male pattern baldness major genetic susceptibility loci AR/EDA2R and 20p11 in female pattern hair loss. Br J Dermatol. 2012; 166:13141318.

17. Redler, S., et al. Investigation of six novel susceptibility loci for male androgenetic alopecia in women with female pattern hair loss. J Dermatol Sci. 2013; 72:186-188.

18. Nuwaihyd, R., et al. Investigation of four novel male androgenetic alopecia susceptibility loci: no association with female pattern hair loss. Arch Dermatol Res. 2014; 306:413-418.

19. Yip, L., et al. Association analysis of oestrogen receptor beta gene (ESR2) polymorphisms with female pattern hair loss. Br J Dermatol. 2012; 166:1131-1134.

20. Redler, S., et al. The oestrogen receptor 2 (ESR2) gene in female-pattern hair loss: replication of association with rs10137185 in German patients. Br J Dermatol. 2014; 170:982-985.

Note from Dr. Bernard Nusbaum: At first glance, Dr. Messenger's article may appear to simply propose nomenclature, but more importantly, it should spark our thought processes regarding the multifactorial nature of female pattern hair loss. In the clinical setting, we recognize that patterns of hair loss in women are not as distinctive as in males. Moreover, our questioning of male hair loss patients generally centers on family history, whereby in women there might be no obvious familial predisposition and we want to know about nutritional status, deficiencies, systemic illnesses, hormonal changes (spontaneous or iatrogenic), toxic exposure, thyroid disease, or other endocrinopathies. I agree with Dr. Messenger that,

$\Rightarrow$ bottom of page 7 


\section{Controversies}

Russell G. Knudsen, MBBS, FISHRS Sydney, Australia drknudsen@hair-surgeon.com

\section{Where Business Meets Medicine}

Hair restoration surgery, being largely cosmetically based and thus discretionary surgery (i.e., not health based), fits at what is often called the "business end of medicine." This is because we physicians actively market ourselves to prospective patients to attract work. As physicians, we are governed by both regulations and ethical standards determined by our local governing authorities and Medical Boards. In some jurisdictions there are also strict rules limiting how physicians may advertise their services. The general purpose is that these rules and ethical standards protect the patients from predatory practices and ensure that physicians practice in their best interests.

Companies that provide products to us have a different set of ethical considerations that influence their marketing or pricing behavior. These more relate to honesty in their advertising/marketing claims. In recent years, pharmaceutical companies have had significant restrictions placed on them in many countries to prevent promotions that reward doctors for using/prescribing their products.

More recently, in our own field, we have seen a couple of companies, new to our market, attempt to grow their business by offering inducements to physicians that reward greater use of their machines. This has caused concern to some physicians who are using their machines.

Incentive-based marketing to physicians causes ethical problems because of the conflict of interest inherent in the proposal. We are supposed to base our recommendations, and treatments, entirely on what is appropriate to the patient's needs and always act in the patient's best interest, not our best interest. We shouldn't have to deal with conflicts of interest between business decisions and patient care decisions. In addition, in many countries and states, incentive-based advertising is illegal if insurance is involved, may violate fraud laws, and is against individual Medical Boards Code of Ethics.

In our field, incentives to utilize a technology would likely be seen to be potentially inducing the quotation of greater graft numbers to our patients than otherwise might be the case, particularly when the incentives are time-limited. In my own practice, I recently purchased an ARTAS ${ }^{\circledR}$ Robotic System and was informed about the "Quick Start Program," which offered me 10,000 free harvest attempts if I completed 30,000 harvest attempts in the first 3 months usage of the machine. I was acutely uncomfortable about this promotion and offered the view that I wouldn't be able to participate because I was not going to base decisions about surgery (strip vs. FUE/Robot) based on an incentive program.

Another recent promotion offered holidays to Hawaii to practices with the most usage of the company's machine in the last 6 months. Both individual physicians and the ISHRS Board of Governors wrote to the company expressing their concerns regarding this type of promotion and, thankfully, in early December, the company involved elected to voluntarily withdraw this promotion.

I believe it is firmly in our best interests to make companies aware that incentive-based advertising/marketing is a poor marketing strategy in our field. Hopefully, the recent events outlined above will help prevent these types of promotions from being offered in the future. in this condition, similar histopathology says nothing about etiology. It is well recognized in dermatology that a distinctive histologic reaction pattern can manifest as a result of a variety of different etiologies and this may very well be true in this setting. Apparently, only certain cases of female hair loss are androgen dependent and "female pattern hair loss" is certainly a better, more inclusive term to describe the multitude of associated factors and clinical presentations seen in women. 